\title{
Composites con refuerzos naturales para aplicaciones en automoción y construcción
}

\author{
C. Elizetxea*, U. Gurmendi* y T. Pijoan*
}

\begin{abstract}
Resumen
La evidencia del agotamiento de las reservas de crudo a nivel mundial junto con el cumplimiento de exigencias medioambientales, cada vez más estrictas, llevan a considerar, cada vez en mayor escala, la necesidad de la utilización de materias primas renovables para el diseño y fabricación de nuevos componentes del vehículo. El objetivo principal de este trabajo se centra en la evaluación de materiales, como la harina de pino y la harina de maíz, para ser utilizados como elementos de refuerzo y ser combinados con diferentes matrices termoplásticas de polipropileno. La definición y puesta a punto de un proceso de compatibilización ha hecho posible la formulación de estas harinas con tres matrices comerciales de base PP. Una posterior selección de material se ha llevada a cabo con el fin de determinar los materiales para la fabricación de una pieza prototipo.
\end{abstract}

\section{Natural reinforced composites for automotive and building applications}

\begin{abstract}
The assumption of the future exhaustion of the crude reserves world-wide and the increasing environmental concerns is leading to consider, more and more insistently, the use of regenerable raw materials for the design and development of new vehicle components. The main objective of this project consists on the evaluation of pine powder and corn powder as reinforcement for different PP resins. A compatilibization study has been defined and set-up in order to combine these two reinforcements with three different resins based on PP. A final material selection has been carried out to produce an automotive prototype part.
\end{abstract}

Keywords

Composites; Natural reinforcements; Corn powder; Pine powder; Automotive.

\section{INTRODUCCIÓN}

El trabajo aquí presentado se enmarca dentro del proyecto europeo Interreg INNOCAP cuyo principal objetivo consiste en evaluar la posibilidad de utilización de harina de pino y harina de maíz, de producción local, como material de refuerzo tanto para aplicaciones de automoción como de construcción.

En comparación con los refuerzos tradicionales (vidrio y carbono principalmente), los refuerzos naturales son menos densos, hecho ampliamente valorado en el sector de automoción, ya que implica la obtención de piezas de menor peso con un menor gasto de combustible y una menor emisión de gases. Además, son muy poco agresivos con los equipos y herramientas de procesado comparadas con las fibras sintéticas. Por otro lado, este tipo de refuerzo presenta buenas propiedades de aislamiento, tanto térmico como acústico, lo que los hace muy interesantes para su utilización en aplicaciones de construcción. Pero, sin duda, uno de los puntos más importantes a tener en cuenta es su positivo impacto medioambiental.

Estos refuerzos naturales aseguran una total reciclabilidad ya que se descomponen, totalmente, sin presentar ningún tipo de residuo tóxico, pudiendo ser eliminados por procesos de biodegradación. Esta propiedad les permite una mejor disposición y aprovechamiento de las piezas elaboradas con estos materiales, una vez terminado su ciclo de vida, posibilitando la aplicación de sistemas de reciclado que no son muy utilizados, actualmente, por las dificultades que acarrean, en este sentido, las fibras de vidrio.

Sin embargo, no todo son ventajas ${ }^{[1-4]}$. La fluctuación a las que están sometidas unida a su naturaleza lignocelulósica hacen necesario realizar investigaciones enfocadas hacia la mejora de su compatibilidad

\footnotetext{
*INASMET-Tecnalia, Paseo de Mikeletegi, 2, 20009 San Sebastián. e-mail: celize@inasmet.es; ugurmend@inasmet.es; apijoan@inasmet.es.
} 
con el polímero, a la disminución de su carácter higroscópico y a la optimización de sus procesos de transformación, aspectos éstos considerados y desarrollados en el marco del proyecto INNOCAP.

\section{MÉTODO EXPERIMENTAL}

\subsection{Definición del plan de trabajo}

Con el objetivo de evaluar la posible utilización de las harinas de pino y de maíz como refuerzos para matrices poliméricas, se han definido las especificaciones para una pieza de automoción interior, no estructural y revestida, y para una pieza exterior para construcción. Este artículo, únicamente se centrará en la aplicación de automoción. Para ello, ha sido necesario un proceso de selección, tanto de los porcentajes de ambos refuerzos naturales, como de las matrices poliméricas a utilizar, el estudio de la compatibilización fibra-matriz polimérica, la preparación de diferentes formulaciones de material compuesto y su caracterización tanto mecánica como física. Por otro lado, se ha procedido a la definición y puesta a punto de los procesos de transformación necesarios para la fabricación de elementos demostradores.

\subsection{Materiales utilizados}

La tabla I resume las principales características de los refuerzos naturales utilizados. Todas las formulaciones se prepararon utilizando un $30 \%$ de refuerzo.

Tabla I. Propiedades refuerzos naturales utilizados

Table I. Properties of used natural reinforcements

\begin{tabular}{lcc}
\hline $\begin{array}{c}\text { Tipo de } \\
\text { Refuerzo }\end{array}$ & $\begin{array}{c}\text { Harina } \\
\text { de Pino }\end{array}$ & $\begin{array}{c}\text { Harina } \\
\text { de Maíz }\end{array}$ \\
\hline Suministrador & $\begin{array}{c}\text { Mont De } \\
\text { Marsant }\end{array}$ & Eurocob \\
Contenido en & & \\
$\quad$ celulosa $(\%)$ & 42,8 & 47,1 \\
Contenido en lignina & 27,2 & 37,3 \\
Distribución media de & & \\
$\quad$ tamaño $(\mu \mathrm{m})$ & 300 & 250 \\
Densidad $\left(\mathrm{g} / \mathrm{cm}^{3}\right)$ & 0,39 & 0,34 \\
\hline
\end{tabular}

Además de los materiales objeto de estudio, harina de pino y harina de maíz, se han estudiado dos refuerzos naturales disponibles comercialmente, C120 y C132, de la firma Rettenmaier, como materiales patrón.

Como matriz polimérica se seleccionó el polipropileno, ya que es una de las matrices mas utilizadas en el sector de la automoción. El estudio se ha realizado con tres tipos de PP cuyas características quedan definidas en la tabla II.

Con el fin de asegurar la correcta adhesión polímero-refuerzo se utilizó, como agente de acoplamiento MAPP (anhídrido maléico, 1\% en peso) POLYBOND 3200 de Chemtura, en diferentes porcentajes.

\subsection{Técnicas de fabricación utilizadas}

Todas las composiciones han sido extruidas en un extrusor bihusillo corrotante, COPERION ZSK 26, indicado para la fabricación de granza termoplástica aditivada. Con el fin de estudiar el efecto que la adición de los diferentes refuerzos ejerce sobre el material base utilizado tradicionalmente, se ha considerado, en cada caso, el PP utilizado como material patrón y sobre él se han realizado los estudios comparativos con diferentes porcentajes de refuerzos y en diferentes condiciones.

\subsection{Técnicas de caracterización utilizadas}

Con la granza obtenida se han inyectado, en una máquina de inyección ARBURG ALL ROUNDER 270 C 300-100, probetas de tracción (norma EN ISO 527) para determinar las propiedades elásticas de los materiales obtenidos y, así, proceder a su comparación. Se han determinado el módulo y la resistencia a tracción, así como la deformación a rotura.

Tabla II. Propiedades de los tipos de PP utilizados

Table II. Properties of used PP grades

\begin{tabular}{lccc}
\hline $\begin{array}{c}\text { Tipo } \\
\text { de PP }\end{array}$ & $\begin{array}{c}\text { PP Natural } \\
\text { 086Y1E }\end{array}$ & $\begin{array}{c}\text { PP } \\
\text { Reciclado }\end{array}$ & $\begin{array}{c}\text { PP/20\%Talco } \\
\text { Reciclado }\end{array}$ \\
\hline Suministrador & REPSOL & ALSER & ALSER \\
MFI (g/10min) & 25 & 9,776 & 8,208 \\
Densidad $\left(\mathrm{g} / \mathrm{cm}^{3}\right)$ & 0,903 & 0,938 & 1,020 \\
\hline
\end{tabular}




\section{RESULTADOS Y DISCUSIÓN}

Tal y como se ha apuntado anteriormente, debido a la diferente polaridad existe entre el la matriz polimérica, PP (apolar), y los refuerzos naturales (polares), es necesaria la utilización de un agente de acoplamiento (MAPP) para facilitar la adhesión entre la matriz y el refuerzo. Con objeto de determinar el porcentaje óptimo de MAPP se prepararon, mediante extrusión, formulaciones con diferentes porcentajes de MAPP. Mediante inyección, se fabricaron probetas de ensayo para determinar su comportamiento en el ensayo de tracción.

La figura 1 muestra los valores obtenidos para el módulo de tracción (MPa), tanto para el PP natural, como para formulaciones reforzadas con un $30 \%$ del producto comercial Rettenmaier C120 y diferentes porcentajes de MAPP, 0, 5, 10 y $15 \%$, respectivamente.

A la vista de los resultados y, teniendo en cuenta además de consideraciones técnicas consideraciones económicas, se determinó el 7 \% como el contenido de MAPP a utilizar en posteriores formulaciones.

Una vez determinado el porcentaje óptimo de MAPP (7\%), se prepararon composiciones de material reforzado, en todos los casos con un 30\% de refuerzo natural (C132, C120, harina de pino y harina de maíz), con las tres matrices de polipropileno seleccionadas (PP natural, PP reciclado y PP/205Talco Reciclado). Sobre todas la formulaciones se determinó su comportamiento a tracción (EN ISO 527), tanto el módulo $(\mathrm{MPa})$ resistencia $(\mathrm{MPa})$ como alargamiento a rotura $(\%)$.

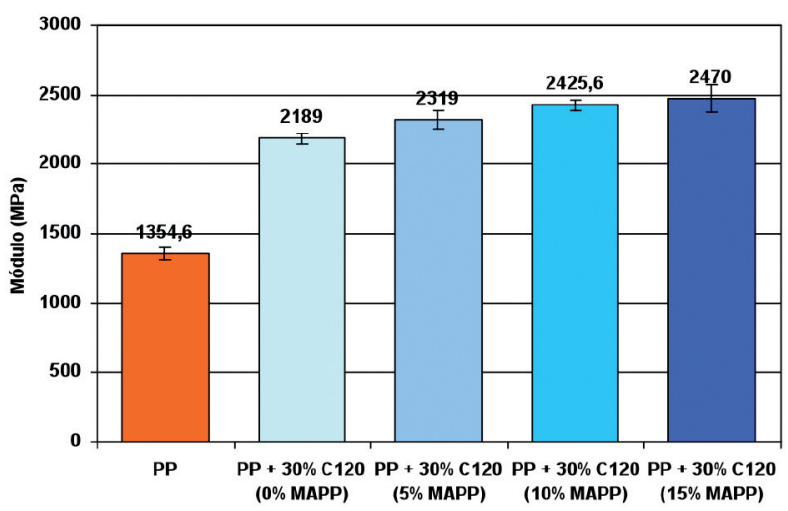

Figura 1. Módulo tracción para formulaciones PP natural+30 \% C120+MAPP (0, 5, 10 y $15 \%)$.

Figure 1. Tensile modulus of PP natural $+30 \%$ C120+MAPP (0, 5, 10 y $15 \%)$ based formulations.
Las figuras 2, 3, 4, 5, 6, 7, 8, 9 y 10 comparan cada una de las propiedades determinadas para cada matriz y tipo de refuerzo.

Puede observarse que en todas las formulaciones fabricadas, independientemente del tipo de refuerzo y de la matriz utilizada, existe un incremento significativo para el valor del módulo de tracción en comparación con el PP sin reforzar utilizado, en cada caso. Cabe resaltar que, en todos los casos, los refuerzos disponibles comercialmente (C120 y C132) presentan valores superiores a los refuerzos naturales desarrollados en el proyecto INNOCAP y que, de entre ellos, el de harina de de pino presenta una mayor

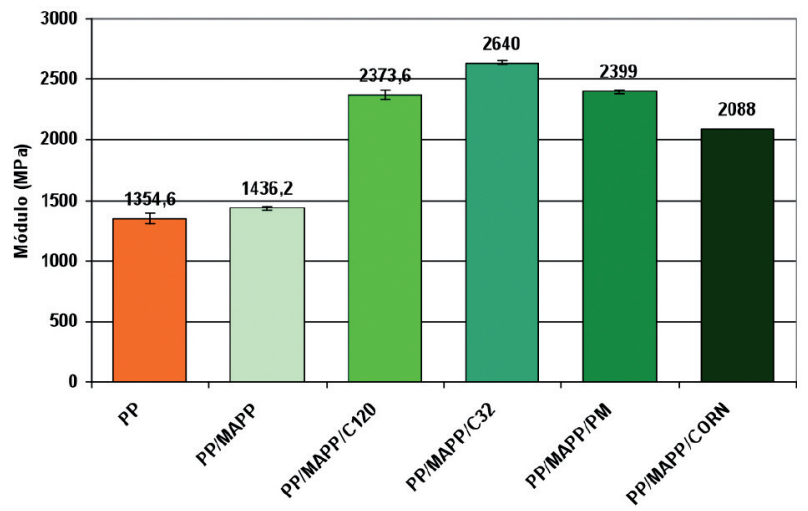

Figura 2. Módulo de tracción formulaciones $30 \%$ refuerzo natural+7 \% MAPP+PP natural.

Figure 2. Tensile modulus of $30 \%$ natural reinforcement+7 \% MAPP+PP natural formulations.

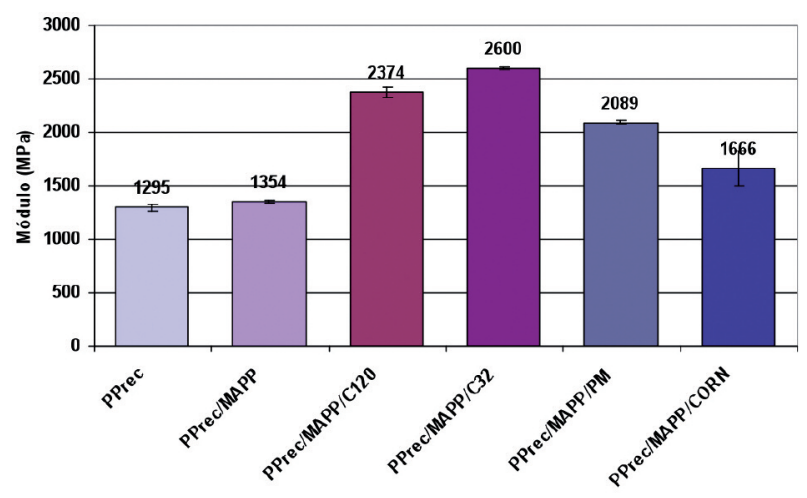

Figura 3. Módulo de tracción formulaciones $30 \%$ refuerzo natural+7 \% MAPP+PP reciclado.

Figure 3. Tensile modulus of $30 \%$ natural reinforcement+7 \% MAPP+recycled PP formulations. 


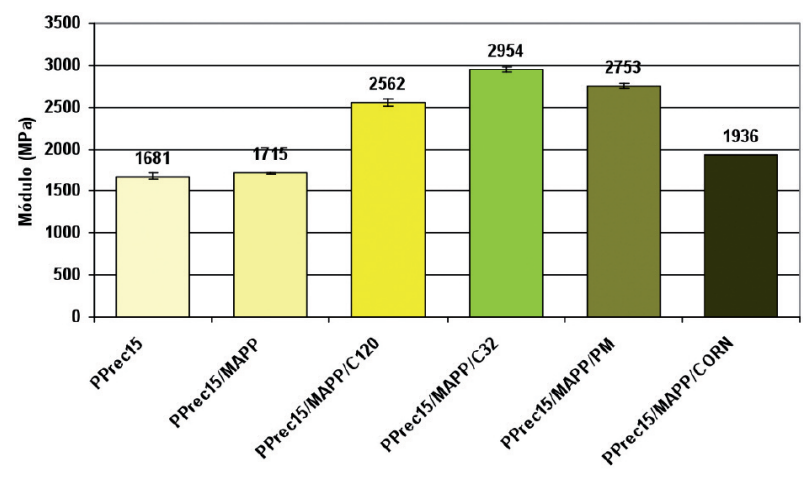

Figura 4. Módulo de tracción formulaciones $30 \%$ refuerzo natural+7 \% MAPP+PP/20 \% talco reciclado.

Figura 4. Tensile modulus $30 \%$ natural reinforcement. $+7 \%$ MAPP $+P P / 20 \%$ talc recycled formulations.

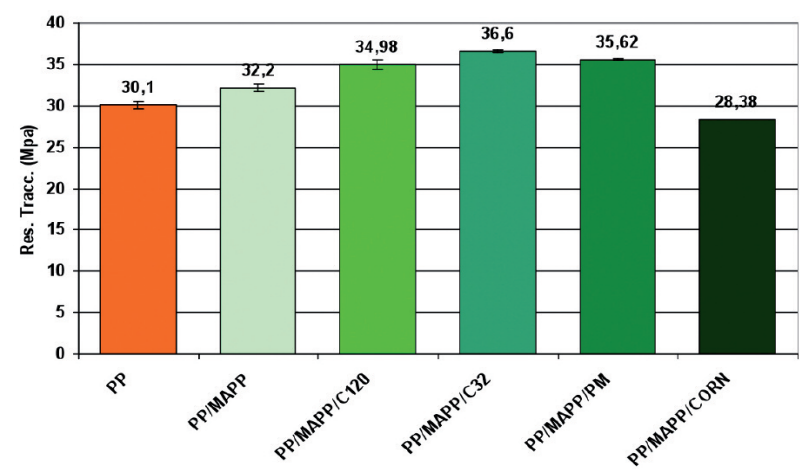

Figura 5. Resistencia a tracción formulaciones $30 \%$ refuerzo natural $+7 \%$ MAPP+PP natural.

Figure 5. Tensile resistance of $30 \%$ natural reinforcement+7 \% MAPP+PP natural formulations.

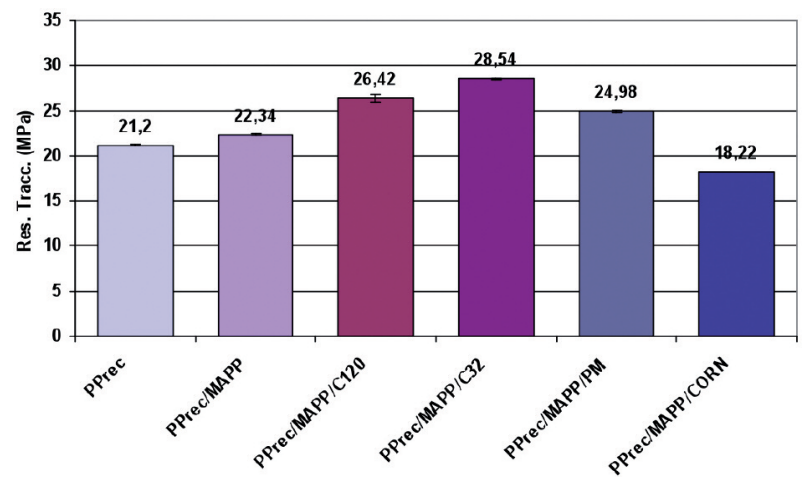

Figura 6. Resistencia a tracción formulaciones $30 \%$ refuerzo natural+7 \% MAPP+PP reciclado.

Figure 6. Tensile resistance of $30 \%$ natural reinforcement+7 \% MAPP+recycled $P P$ formulations.

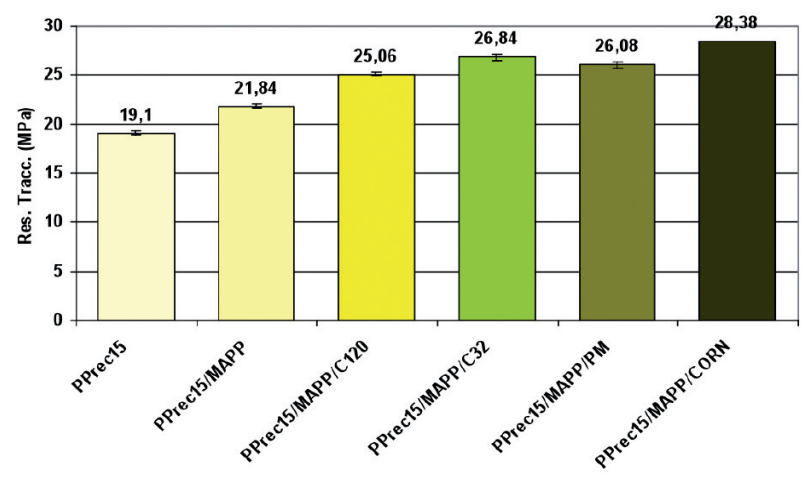

Figura 7. Resistencia a tracción formulaciones $30 \%$ refuerzo natural+7 \% MAPP+PP/20\% talco reciclado.

Figure 7. Tensile resistance of $30 \%$ natural reinforcement+7 \% MAPP+PP/20\% talc recycled.

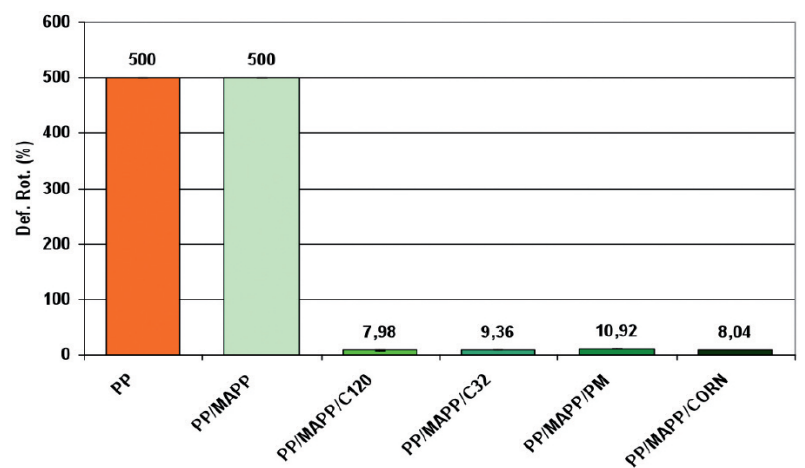

Figura 8. Alargamiento a rotura formulaciones $30 \%$ refuerzo natural+7 \% MAPP+PP natural.

Figure 8. Elongation at break of $30 \%$ natural reinforcement+7 \% MAPP+PP natural formulations.

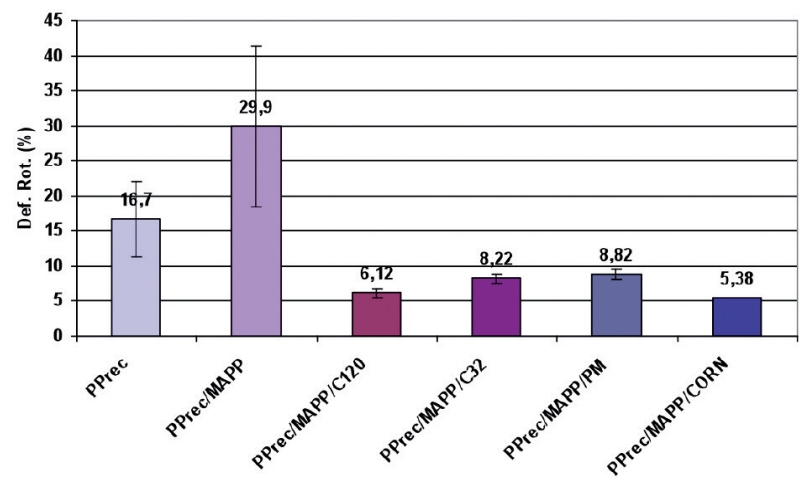

Figura 9. Alargamiento a rotura formulaciones $30 \%$ refuerzo natural+7 \% MAPP+PP reciclado.

Figure 9. Elongation at break of $30 \%$ natural reinforcement $+7 \%$ MAPP+recycled $P P$ formulations. 


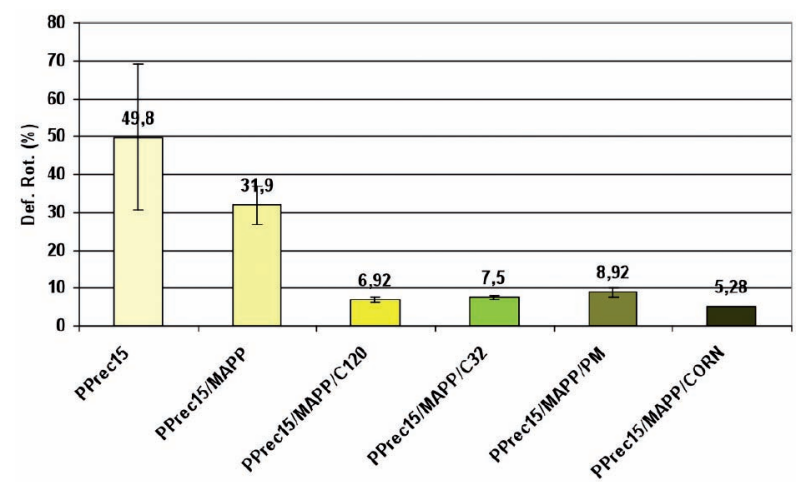

Figura 10. Alargamiento a rotura formulaciones $30 \%$ refuerzo natural+7 \% MAPP+PP/20\% talco reciclado.

Figure 10. Elongation at break of $30 \%$ natural reinforcement+7 \% MAPP $+P P / 20 \%$ talc recycled.

capacidad de refuerzo que el de harina de maíz. Este mismo comportamiento se observa para la resistencia a tracción. En cuanto al alargamiento a rotura, éste se ve limitado de manera muy importante ya que se obtiene un material mucho más rígido que el PP original.

Comparando los resultados de todas las formulaciones estudiadas, puede concluirse que el PP natural y el reciclado con un $20 \%$ de talco, presentan valores superiores al PP reciclado.

Teniendo en cuenta las especificaciones definidas inicialmente para una pieza de automoción interior, no estructural y revestida como, por ejemplo, el soporte de altavoz (Fig. 11), estos materiales, tanto el PP natural como el PP/20 \% talco reciclado, pueden considerarse como materiales candidato para su fabricación.

\section{CONCLUSIONES}

- Los refuerzos naturales de harina de pino y de maíz desarrollados en el proyecto INNOCAP presentan propiedades mecánicas comparables a los productos comerciales también estudiados.

- Es necesario adaptar los procesos de fabricación convencionales de plásticos, intentando trabajar en las condiciones menos agresivas para mantener

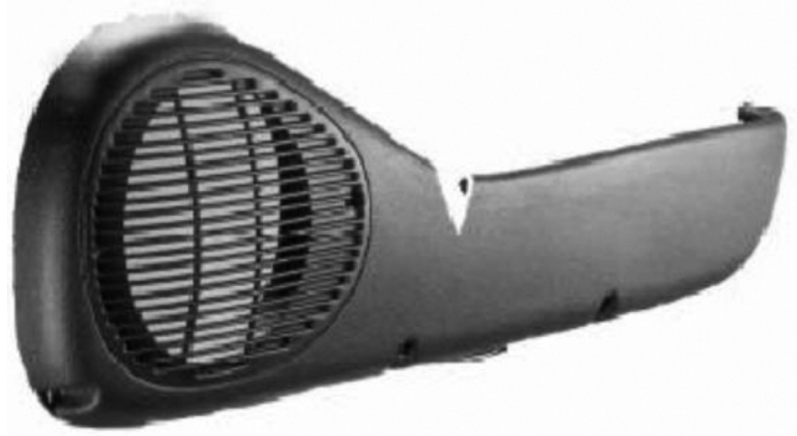

Figura 11. Soporte de altavoz.

Figure 11. Door inner pocket.

y preservar las propiedades de los refuerzos naturales. Es necesario evitar procesos de degradación por tiempos de permanencia superiores a $1 \mathrm{~min}$, a temperaturas por encima de $\operatorname{los} 180^{\circ} \mathrm{C}$.

- Los materiales desarrollados cumplen con los requerimientos definidos para una aplicación genérica de automoción interior, no estructural y revestida.

\section{Agradecimientos}

Este trabajo ha sido financiado por el proyecto Interreg IV A, POCTEFA EFA14/08, INNOCAP, y ha contado con la participación de, además de INASMET-Tecnalia, CANBIO, CDAPP y NOBATEK.

\section{REFERENCIAS}

[1] M. Münker, R. Holtmann y W. Michaeli, KU Kunststoffe Plast Europe 89 (1999) 29-31.

[2] N.A. Marcovich, M.M. Reboredo y M.I. Aranguren J. Appl. Polym. Sci. 68 (1998) 2.069-2.076.

[3] T. Reubmann, R. Mieck y R. Bayer, KU Kunststoffe Plast Europe 89 (1999) 23-26.

[4] C. Elizetxea, A. Rubio, N. Calleja e I. Gaztelumendi, Proc. Global Symp. Recycling, Waste Treatment Clean Technol., REWAS 2004, vol. I, Madrid, España, Ed. INASMET, 2004, pp. 423 432. 